\title{
Are Learning Styles Relevant To Virtual Reality?
}

\author{
Chwen Jen Chen \\ Universiti Malaysia Sarawak \\ Seong Chong Toh \\ Wan Mohd Fauzy Wan Ismail \\ University Science Malaysia
}

\begin{abstract}
This study aims to investigate the effects of a virtual reality (VR)-based learning environment on learners with different learning styles. The findings of the aptitude-by-treatment interaction study have shown that learners benefit most from the VR (guided exploration) mode, irrespective of their learning styles. This shows that the VR-based environment offers promise in accommodating individual differences in terms of learning style. In addition, the significant positive effect of the $V R$ (guided exploration) mode-which provides additional navigational aids-over the VR (non-guided exploration) mode-which does not provide additional navigational aids - also implies the importance of providing VR-based learning environments with proper instructional design to achieve the desired educational outcomes. (Keywords: virtual reality, learning style, aptitude-by-treatment interaction, learning environment.)
\end{abstract}

Aptitude-by-treatment interaction (ATI) research investigates the effects of learner aptitudes and traits on learning outcomes from different forms of instruction (Berliner \& Cahen, 1973; Cronbach \& Snow, 1969). The major assumption of this kind of research is that it is possible and desirable to adapt the nature of instruction to accommodate individual differences in terms of ability, style, or preference to improve learning outcomes. Interactions occur between aptitudes and treatments when individual differences predict different outcomes from alternative forms of structural or presentational properties. Tobias (1981) has given a clear elaboration to help in understanding the concept of interaction, which is summarized below.

In Figure 1 (page 124), the $\mathrm{x}$-axis represents any individual difference measure, while the $y$-axis represents instructional outcomes. The functions, also known as regression slopes, in the figure represent the results for two different instructional treatments, A and B. This figure indicares that learners with low scores on the aptitude measure also perform poorly on the instructional outcome measure under treatment $A$. However, learners with similar low scores on the individual difference measure do quite well on the outcome measure when they are given treatment $\mathrm{B}$. The contrary result is observed for learners with high scores on the aptitude measure. The ATI interaction in Figure 1 is a disordinal interaction. This means that not only the regression slopes are different, but also they are intersected (Jonassen \& Grabowski, 1993). Such disordinal interactions are useful for appropriately assigning learners to different instructional treatments or methods.

Another type of ATI interaction is called ordinal interaction, where one treatment produces equal or better results for all learners within the range of 